\title{
A Novel Oblique Detonation Structure and Its Stability *
}

\author{
TENG Hong-Hui(滕宏辉), ZHAO Wei(赵伟), JIANG Zong-Lin(姜宗林) \\ Key Laboratory of High Temperature Gas Dynamics, Institute of Mechanics, Chinese Academy of Sciences,
} Beijing 100080

\section{(Received 22 March 2007)}

\begin{abstract}
Oblique detonation structures induced by the wedge in the supersonic combustible gas mixtures are simulated numerically. The results show that the stationary oblique detonation structures are influenced by the gas flow Mach number, and a novel critical oblique detonation structure, which is characterized by a more complicated wave system, appears in the low Mach number cases. By introducing the inflow disturbance, its nonstationary evolution process is illustrated and its stability is verified.
\end{abstract}

PACS: 47.40. $-\mathrm{x}, 47.85 . \mathrm{Gj}, 82.40 . \mathrm{Fp}$

Detonation waves are one kind of supersonic combustion induced by strong shock and sustained by the post-shock rapid heat release. It concerns the nonlinear coupling of the shock wave and the combustion, embarrassing intensive investigation. In recent years, the potential applications of detonation waves in future hypersonic propulsion systems have been widely studied. There are two kinds of detonation propulsion configurations generally, one is the pulsed detonation engine, ${ }^{[1]}$ the other is the ram accelerator. ${ }^{[2]}$ In the latter configuration, the wedge in the supersonic gas flow generates oblique shock waves and the shock ignites the combustible inflow mixtures, which will induce the oblique detonation wave downstream. To achieve oblique detonation propulsion, its structure and stability must be first clarified. The sketch of the physical model is shown in Fig. 1(a) and the sketch of the oblique detonation structure from previous investigations ${ }^{[3,4]}$ is shown in Fig. 1(b), whose coordinates are rotated along the wedge. It can be observed that the oblique shock and detonation waves are jointed by an inflexion, generating the oblique detonation structure together with the deflagration wave beneath the inflexion. Recently, the stability of this structure is investigated by the advanced numerical methods, and various factors which influence the oblique detonation structure, such as the pressure, temperature, inflow gas Mach number, are demonstrated..$^{[5,6]}$ However it is still very difficult to realize stable oblique detonation waves in experiments, which indicates the necessity of further investigation on the oblique detonation structure.

In this Letter, a novel detonation structure is illustrated using the detailed chemical reaction model and the adaptive unstructured mesh technology, and further its stability are investigated. The computational simulation is carried out in the dashed zone shown in Fig. 1(a), and the coordinate is rotated to the direction along the wedge surface. The viscosity effects on the structure and its stability have been discussed numerically ${ }^{[7]}$ and theoretically. ${ }^{[8]}$ Both the results demonstrate that the effects are limited in a very thin boundary layer and have little influence on the main wave structure. Therefore the governing equations can be simplified into Euler equations. Adaptive unstructured quadrilateral grid ${ }^{[9]}$ is adopted and the MUSCL-Hancock scheme is applied with the HLLC solver, ${ }^{[10]}$ which has the second space accuracy. The combustion model is vital in the detonation simulation and recent numerical results show that it will influence the stability of detonation waves. ${ }^{[11]}$ Different from the usual single irreversible Arrhenius reaction model, the hydrogen/air chemical reaction model is selected from the widely used CHEMKIN package, where 11 species $\left(\mathrm{H}_{2}, \mathrm{O}_{2}, \mathrm{O}, \mathrm{H}, \mathrm{OH}, \mathrm{HO}_{2}, \mathrm{H}_{2} \mathrm{O}_{2}, \mathrm{H}_{2} \mathrm{O}, \mathrm{N}_{2}\right.$, $\mathrm{N}, \mathrm{NO}$ ) and 23 reactions are account for in chemical reactions. The stiff problem due to chemical reaction calculation is solved by the DVODE package. ${ }^{[12]}$ This set of codes has been verified by many simulations and some meaningful results have been achieved. In this case, the computational region is $11 \mathrm{~mm} \times$ $5 \mathrm{~mm}$ and the wedge length is $10 \mathrm{~mm}$. The slip reflecting boundary condition is used on the wedge surface and the other boundaries are interpolated under the assumption of the zero first-order derivatives of all flow parameters. The initial condition is hydrogen/air premixed gas with $\mathrm{H}_{2}: \mathrm{O}_{2}: \mathrm{N}_{2}=2: 1: 4$, temperature $298.15 \mathrm{~K}$, and pressure $101325 \mathrm{~Pa}$. The wedge angle is $30^{\circ}$ and the initial gas velocity is decided by the given Mach number, which is changed case by case in our simulation. The grid is generated by the GAMBIT software package, with the initial homogeneous scales $0.5 \mathrm{~mm} \times 0.5 \mathrm{~mm}$. In the computation, the grid adaptation divides one quadrilateral grid into the same four grids near the shock and counter surface. Several adaptation levels are tested to eliminate the grid effect: with the four level adaptation the structure will appear upstream and the wave system is blurred, but 
the five-level simulation shows the same wave structure as the six-level simulation. Therefore the fivelevel adaptation is used and the finest grids can be adapted to $0.016 \mathrm{~mm} \times 0.016 \mathrm{~mm}$ in this simulation.

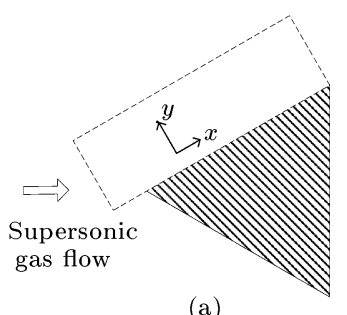

(a)

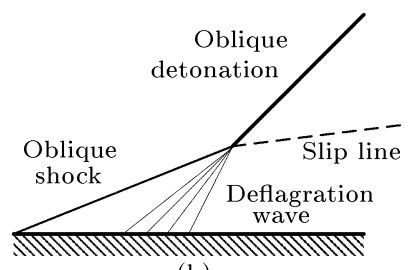

(b)
Fig. 1. Sketches of the physical model and the oblique detonation structure.
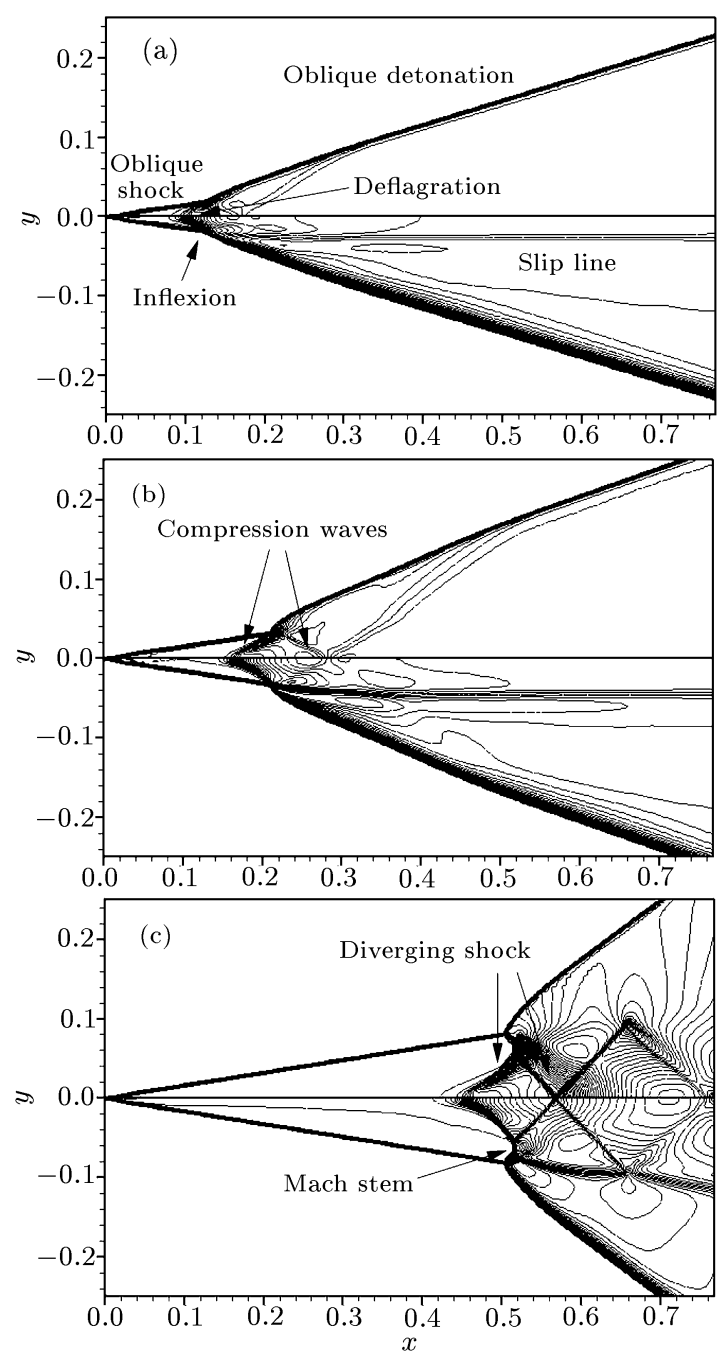

Fig. 2. Pressure (upper) and temperature (lower) of oblique detonation wave structures: (a) $M a=8.0$, (b) $M a=7.5$, (c) $M a=7.0$.

The oblique detonation wave structures with the gas-flow Mach numbers $M a=8.0,7.5$, and 7.0 are shown in Fig. 2 using the pressure and temperature contours. It can be found that the structures are influ- enced by the inflow-gas $M a$ greatly. For the higher $M a$ as shown in Fig. 2(a), the structure is similar to that shown in Fig. 1(b). This structure surface is composed of two parts, the upstream oblique shock wave and the downstream oblique detonation wave with larger inclination rates, which is connected by an inflexion. The inflexion on the wave surface is actually the joint points among the oblique shock wave, oblique detonation wave and the deflagration wave. A slip line, which is from the inflexion and extends downstream, can be observed due to the different gas density and velocity across the detonation and deflagration waves. This case also demonstrates that the simulation is credible and can be used to study the oblique detonation structure. For the middle $\mathrm{Ma}$ as shown in Fig. 2(b), the inflexion location moves downstream and the inclination rate of the oblique detonation wave becomes larger. Meanwhile the wave system beneath the inflexion becomes more different: there are two compression waves jointing at the inflexion. The upstream one lays over the deflagration wave, and the downstream one reflects between the wall and the slip line, which has the wall reflection point at about $x=0.28$. For the lower $M a$ as shown in Fig. 2(c), the inflexion location moves downstream further and the inclination rate of the oblique detonation wave also rises, while a characteristic wave structure is involved around the deflagration wave surface. A Mach stem including two diverging shock waves, which compose the $\lambda$ shock,
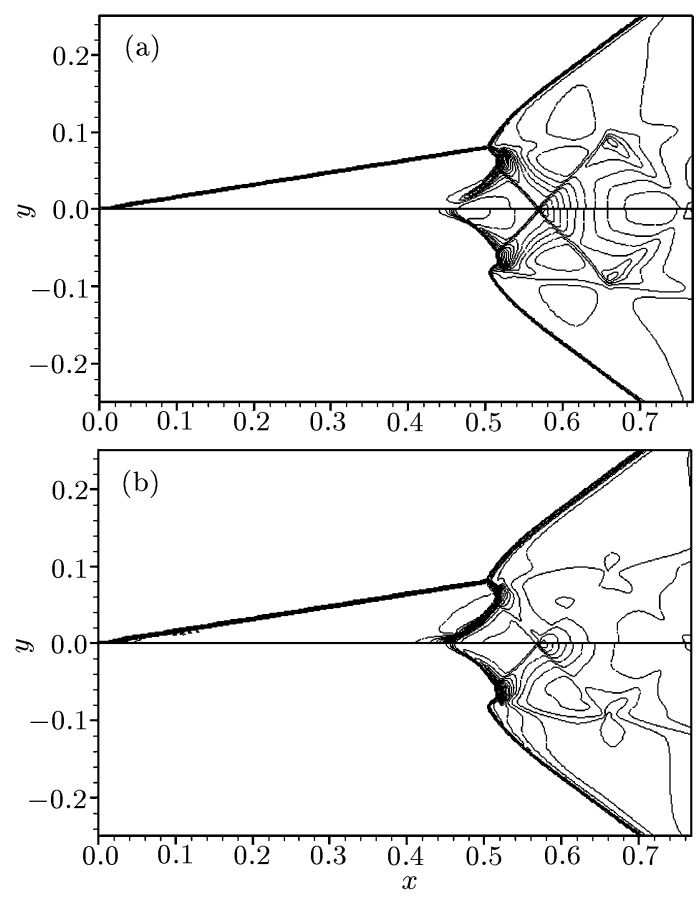

Fig. 3. Densities of the oblique detonation wave structure with $M a=7.0$ : (a) total (upper) and $\mathrm{H}_{2} \mathrm{O}$ (lower), (b) $\mathrm{H}_{2}$ (upper) and $\mathrm{OH}$ (lower). 

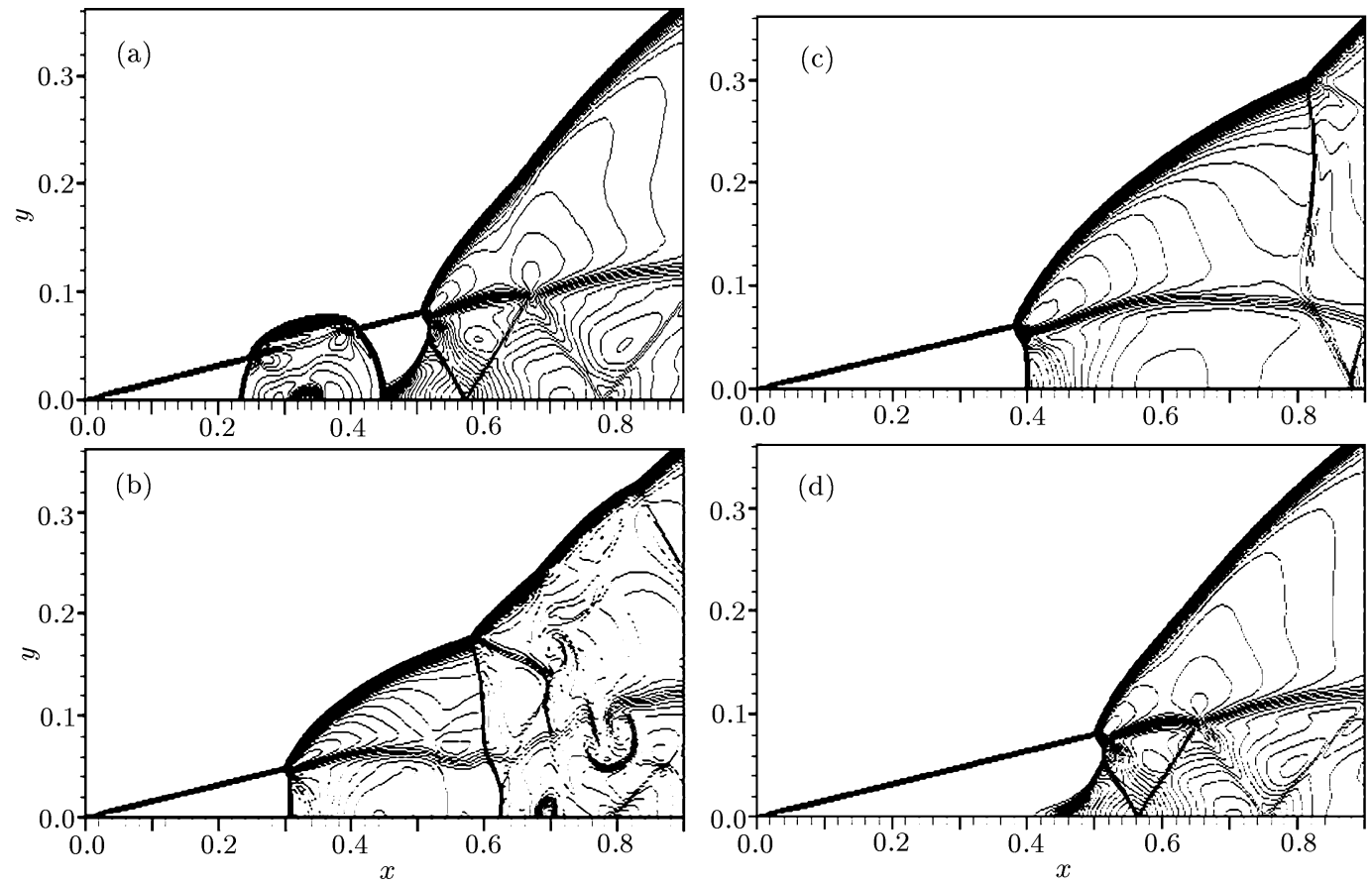

Fig. 4. Time sequences of temperature contours of the critical oblique detonation wave structure with the disturbed inflow density ratio $\mathrm{H}_{2}: \mathrm{O}_{2}: \mathrm{N}_{2}=0: 1: 4$ at different times: (a) $1.6^{\mu} \mathrm{s}$, (b) $3.3^{\mu} \mathrm{s}$, (c) $6.9^{\mu} \mathrm{s}$, (d) $18.6^{\mu} \mathrm{s}$.
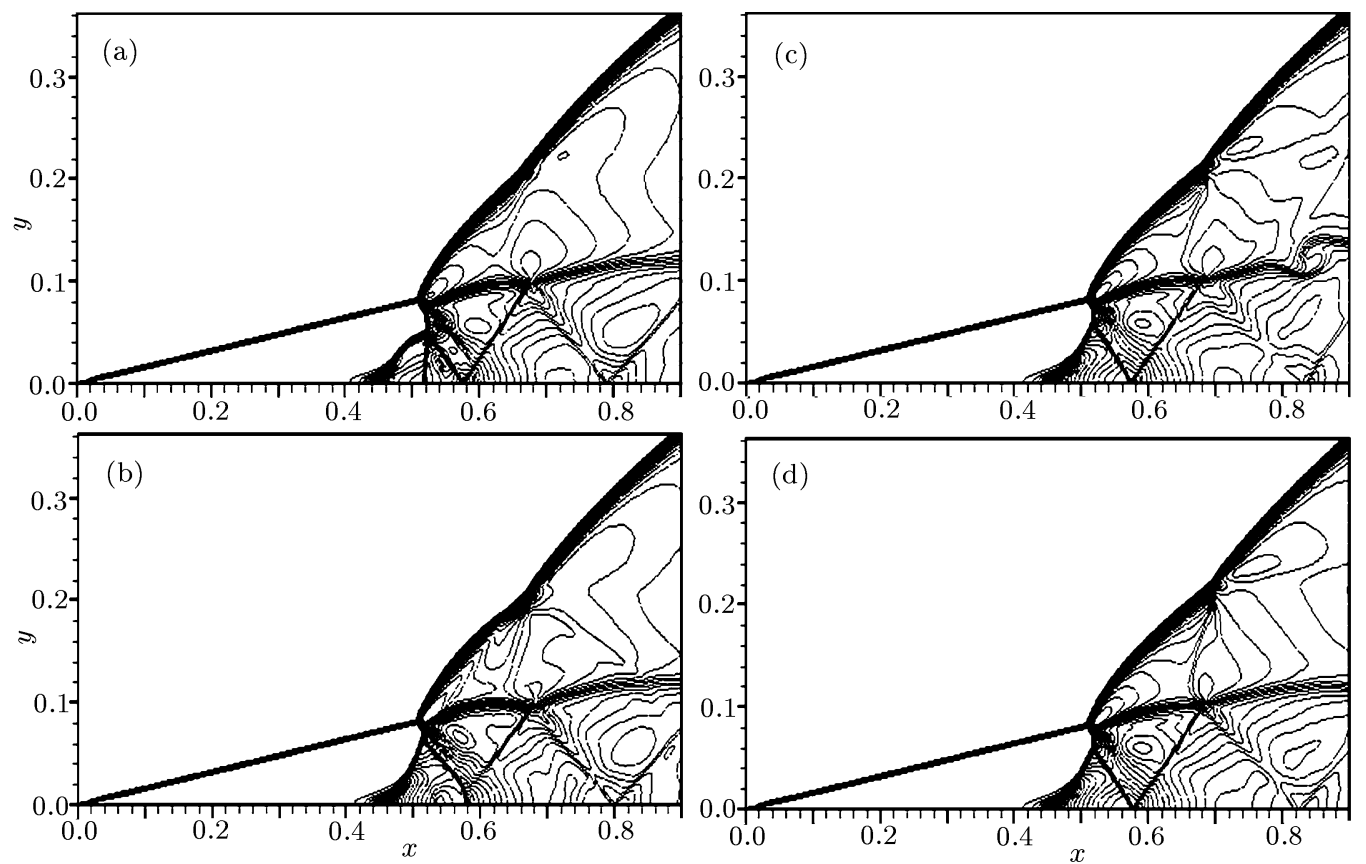

Fig. 5. Time sequences of temperature contours of the critical oblique detonation wave structure with the disturbed inflow density ratio $\mathrm{H}_{2}: \mathrm{O}_{2}: \mathrm{N}_{2}=2: 1: 2$ at different times: (a) $1.8^{\mu} \mathrm{s}$, (b) $3.4^{\mu} \mathrm{s}$, (c) $4.9^{\mu} \mathrm{s}$, (d) $6.8^{\mu} \mathrm{s}$.

appears beneath the inflexion. This $\lambda$ shock is similar to that appearing in the shock and the boundary layer interaction, which has been studied widely. ${ }^{[13]}$ However, they must have different mechanisms because this is a stationary structure concerning the coupling of the shock and combustion. According to the previous detonation research, it can be called the critical oblique detonation wave structure.

Figure 3 shows the specie density distribution of the oblique detonation structure corresponding to Fig. 2(c), with the gas-flow $M a 7.0$. It can be observed that the $\lambda$ shock has the different coupling patterns in the combustion region. The combustion is initiated immediately behind the upstream diverging shock wave and the Mach stem, while the downstream diverging shock wave locates in the combustion productions. The upstream diverging shock wave lays over the deflagration surface and it becomes weaker 
gradually near the wall. This is easy to be understood because the shocked gas near the wall has experienced the longer induction time, so it can selfignite. To the shocked gas away from the wall, it experiences the shorter induction time before entering the combustion zone, so the ignition must be achieved with the help of the upstream diverging shock wave. Therefore the strength of upstream diverging shock wave rises gradually when its distance from the wall rising. To the shocked gas across the oblique shock near the inflexion, the upstream diverging shock is so weak that a stronger Mach stem, which is actually a normal shock, has to form instead of the oblique diverging shock. Meanwhile, the downstream diverging shock wave, which locates in the combustion products and reflects repeatedly between the wall and the slip line, and forms to adjust the gas parameters of the combustion products. Therefore the characteristic $\lambda$ shock wave forming is for matching the different induction times of the shocked gas. If the oblique shock wave is weak, the shocked gas near the inflexion will need the Mach stem to ignite so this structure appears. The structure shown in Fig. 2(b) can be regarded as a simplified one of this structure, but the higher gas $M a$ makes the upstream diverging shock strong enough so that the Mach stem is unnecessary. This structure will form downstream in the case of $M a=6.9$ and it cannot appear in the computational zone in the case of $M a=6.8$. The wedge angle also influences the oblique detonation structure forming greatly. For a larger wedge angle case, the oblique shock is stronger, giving the same gas Mach number, so it is unnecessary to form the characteristic $\lambda$ shock wave. For a smaller wedge angle case, this structure will move downstream but it can be observed clearly in higher Ma cases. For example, a clear Mach stem can be observed first in the case of the wedge angle $30^{\circ}$ at $M a=7.2$, while the wedge angle $28^{\circ}$ at $M a=7.4$.

The stability of the oblique detonation wave structure is very important in the application. By introducing the density disturbance in the inflow gas, the time sequences of temperature contours of this critical structure are shown in Figs. 4 and 5. A disturbance region, which has the same parameters as the other inflow gas expect the specie densities, is first located the zone $-0.05<x<0$ and $0<y<0.05$ before the critical structure shown in Fig. 3 . The disturbed specie densities of Figs. 4 and 5 are set be $\mathrm{H}_{2}: \mathrm{O}_{2}: \mathrm{N}_{2}=0: 1: 4$ and $\mathrm{H}_{2}: \mathrm{O}_{2}: \mathrm{N}_{2}=2: 1: 2$, respectively. Generally, the numerical results of both cases demonstrate the stabil- ity of the critical structure. In the first case, the reactions occur earlier and disturb the flow field seriously, with a new reaction interface forms upstream, as shown in Figs.4(a) and 4(b). However, this interface is unsteady and it will move downstream, as shown in Fig. 4(c). In the end the same critical structure reappears in the original position, as shown in Fig. 4(d). The disturbance introduced in Fig. 5 operates near the deflagration wave, and then the downstream wave structure is influenced. It can be observed that the oblique detonation wave and the slip line are curved, as shown in Figs. 5(b) and 5(c), but the structure near the inflexion keeps nearly constant and the downstream flow field re-stabilizes soon. The above methods have introduced the disturbances in the induction and reactive zones separately, but the structure returns to the initial configuration. Therefore, it can be concluded the critical oblique detonation structure is stable in this simulation.

In summary, we have numerically studied the oblique detonation structure induced by the wedge in the supersonic combustible gas mixtures. It is found out that the structure is influenced by the incident Mach number and there is a novel oblique detonation structure in the low Mach number case. This novel structure referred to as the critical oblique detonation structure has complicated wave systems beneath the inflexion, concerning the coupling of the shock and the deflagration wave. By introducing the inflow disturbance, this structure is verified to be stable and resistable to the inflow disturbance, which favours its potential applications.

\section{References}

[1] Roy G D et al 2004 Prog. Energ. Combust. 30545

[2] Nettleton M A 2000 Shock Waves 109

[3] Li C, Kailasanath K and Oran E S 1994 Phys. Fluids 6 1600

[4] Papalexandris M V 2000 Combust. Flame 120526

[5] Fusina G, Sislian J P and Parent B 2005 AIAA J. 431591

[6] Fernando L, Silva F D and Deshaies B 2000 Combust. Flame 121152

[7] Li C, Kailasanath K and Oran E S 1993 AIAA Paper 930450

[8] Daimon Y and Matsuo 2004 Sci. Technol. Energ. Mater. 65111

[9] Sun M and Takayama K 1999 J. Comput. Phys. 150143

[10] Toro E F 1999 Riemann Solvers and Numerical Methods for Fluid Dynamics (Berlin: Springer)

[11] Ng H D and Lee J H S 2003 J. Fluid Mech. 476179

[12] Brown P N et al 1989 SIAM J. Sci. Stat. Comput. 10 1038

[13] Weber Y S et al 1995 Phys. Fluids 72475 